\title{
Synergic Fabrication of Naringin Molecule into Polymeric Nanoparticles for the Treatment and Nursing Care of Lung Cancer Therapy
}

\section{Lingqiao Yan}

First People's Hospital of Wenling

Hui Chen

First People's Hospital of Wenling

Mindan Xie ( $\square$ mindan.x@yahoo.com )

First People's Hospital of Wenling https://orcid.org/0000-0001-5464-9268

\section{Research Article}

Keywords: Supramolecular, Naringin, Lung cancer, Apoptosis, Hemocompatibility.

Posted Date: March 11th, 2021

DOI: https://doi.org/10.21203/rs.3.rs-255282/v1

License: (c) This work is licensed under a Creative Commons Attribution 4.0 International License.

Read Full License 


\section{Abstract}

Lung cancer is the third most common cause of death and the main factor of cancer-related deaths in both males and females in the United States to the rest of the world. Diagnosis at an important level is associated with high mortality in the population of cases. Herein, we present a very easy and costeffective method that incorporates drugs reconstruction, tumor-specific targeting supramolecular nanoassembly, and therapeutically to overcome the different challenges raised by the distribution of the pharmaceutical potential anticancer drug Naringin. On covalent conjugations of hydrophobic linoleic acid by hydroxyl group, the Naringin prodrugs were skilled in impulsively nanoassembly into extremely steady nanoparticles size ( 100 nm). Electron microscopic techniques have verified the newly synthesized morphology of Naringin-NPs. The anticancer properties of Naringin and Naringin-NPs against A549 and HEL-299 (lung carcinoma) cancer cell lines have been evaluated after successful synthesis. Other research, such as dual staining acridine orange/ethidium bromide, Hoechst 33344 and flow cytometry study on the apoptosis mechanisms have shown that proliferation in lung cancer cells is associated with apoptosis. Compared to Naringin, Naringin-NPs demonstrate excellent biocompatibility, this study clarified the Naringin-NPs as a healthy and positive lung cancer treatment and care chemotherapeutics technique and deserve further clinical evaluations.

\section{Highlights}

1. Fabrication of Naringin on covalent conjugations of linoleic acid by impulsively nanoassembly (Naringin-NPs).

2. The in vitro cytotoxicity activity shows Naringin-NPs induced apoptosis in human lung cancer cells.

3. The morphological examinations and cell death of Naringin-NPs have studied AO/EB and nuclear staining methods.

4. The cell death of Naringin-NPs was confirmed by flow cytometry analysis.

5. Naringin-NPs shows excellent hemocompatibility profiles.

\section{Introduction}

Lung cancer remains the leading cause of cancer-related mortality in the United States, with a quarter of all tumour deaths due to this condition in 2015. A large number of patients are commonly diagnosed with advanced non-small cell lung cancer (NSCLC) owing to its asymptomatic form, requiring intensive and precise care following diagnosis [1-4]. Combination drug treatments are being investigated, as the synergistic activity of several medications may theoretically lead to improved clinical effectiveness and decreased risk for developing drug resistance in cancer cells [5-8]. Gemcitabine-platinum and Cisplatingemcitabine-bevacizumab are some of the drug formulations currently under investigation for advanced NSCLC therapy. Gemcitabine, an FDA-approved chemotherapy compound, has also been developed for first-line chemotherapy with NSCLC that has been used in clinical studies in conjunction with radiotherapy [9-12]. However, these modern therapies are constrained by quick relapse, toxic effects, and 
non-specific distribution of the medication formulations included, resulting in poor clinical effectiveness. Surprisingly weak survival rates in Patients treated can also be related to an innate susceptibility to chemotherapy due to the improved capacity of cancer cells to regenerate DNA damage after radiation therapy (RT). Thus it is important to build a method that can resolve above that the limitations and provide selective and controlled releases of these therapeutic reagents for successful chemo-radiation therapy in the care of these patients with lung cancer [13-16].

The numbers of immunological trials demonstrated the scope for prevention and treatment of dietary phenolic and flavonoids [17-20]. Flavonoids are probably involved in reducing tumorigenesis by preventing DNA damage (induction), suppressing tumor growth (promotion) and regressing tumor invasion (proliferation). Anticancer properties of flavonoids have been identified in eukaryotic cell types by specific cytotoxicity, antiproliferative activity and induction of apoptosis [21-24]. Naringin is also one of the found naturally flavonones found in citrus and grapes fruit. Naringin shows a broad variety of pharmacological properties such as anti-atherogenic, anti-inflammatory, anti-cancer, hepatoprotective and anti-mutagenic, with minimal to no toxic effect. Naringin has hydroxyl groups in its benzene ring that are accountable for its potent antioxidant activity and extensive physicochemical effects [25-28].

In addition, unperformed modification of Naringin to inactive metabolites correspondents may be significantly inhibited after systemic control due to reduced metabolic properties of in the plasma membranes. Therefore, the Naringin encapsulation prodrug was shown to reduce drugs stimulations and increase the performance of drug loading efficacy by the effects of permeability and retention of enhancement [29-33]. In this report, we defined simple and superior method of effect that energetically combine with the supramolecular nanoassembly tumor targeting and drug reconstruction therapies of Naringin. To effectively target the objective, hydrophobic linoleic acid (LA) is an integral fatty acids, by hydroxyl group formation, was conjugated with the amino moiety of the Naringin equivalent. Fruitfully, we discovered in the water solutions the Naringin prodrugs of increasing self-modeling into the nanoparticles of nanoassembly. The in vitro proliferation of lung cancer cells was investigated by the nanoassembly Naringin prodrug and the morphological deviations and cell death examinations of lung cancer cells were identified.

\section{Experimental Part}

\subsection{Fabrication of Naringin-NPs}

DIEA was combined with Naringin (75 mg, $0.20 \mathrm{~mol})$ and hydrophobic linoleic acid $(78 \mathrm{mg}, 0.20 \mathrm{~mol})$ solutions containing $2 \mathrm{~mL}$ of DMF (103 mg, $8.1 \mathrm{~mol})$. The reaction mixture solutions was allowable to stir overnight at $37^{\circ} \mathrm{C}$ and then to evaporate to extract the DMF solution. The reaction mixtures that have been absorbed in the $\mathrm{DCM}$. Five percent citric acid, $\mathrm{NaCl}$ and aqueous $\mathrm{NaHCO}_{3}$ is used to wash the organic coating. The DCM solution were removed using vacuum dried with $\mathrm{Na}_{2} \mathrm{SO}_{4}$ solution. Figure 1 demonstrates the comprehensive fabrication techniques. 


\subsection{Characterization}

The morphology of Naringin-NPs was tested by transmission electron microscopy (TEM; JEM-1200EX, Japan) ensuing with phosphotungstic acid staining $(2 \%, w / v)$ for $3 \mathrm{~min}$, and Naringin-NPs were then perceived and examined by employing them on films of copper grid-irons. The polydispersity index (PDI), zeta potential and particle size of nanoparticles were examined using the Zetasizer ZEN3600 (Malvern, England).

\subsection{Cell culture}

A549 and HEL-299 lung cancer cell lines were cultured in DMEM (Gibco, NY, USA) enclosing 10\% fetal calf serum (Hyclone, Logan, UT, USA), $100 \mu \mathrm{g} / \mathrm{mL}$ streptomycin (Life Technology, NY, USA), $100 \mathrm{IU} / \mathrm{mL}$ penicillin (Bioscience Technology, NY, USA), 1\% L-glutamine (Bioscience Technology), and 1\% nonessential amino acids (Bioscience Technology) at $37^{\circ} \mathrm{C}, 95 \%$ and $5 \%$ for humidity and $\mathrm{CO}_{2}$ respectively.

\subsection{In vitro proliferation assay by MTT}

According to previously recorded protocols [34-36], a MTT assay was achieved. It is used to measure Naringin and Naringin-NPs for in vitro cytotoxicity. The cells of A549 and HEL-299were plated $2 \times 10^{4}$ cell per well in a 96-well culture plate for the attachment followed by cultured for $24 \mathrm{~h}$ at $37^{\circ} \mathrm{C} .10 \mu \mathrm{L}$ of the MTT solution was applied to each well after incubation at $37^{\circ} \mathrm{C}$ for 24 hours, and the $525 \mathrm{~nm}$ absorbance was analyzed $2 \mathrm{hrs}$ later by Eliza microplate reader (TECAN Sunrise, Switzerland). The percentage of the cell proliferation were calculated by using the graph pad prism software. As seen below, the cell viability (percent) was evaluated using the formula;

$\%$ of cell viability $=O D_{\text {treated }} / O D_{\text {control }} \times 100$

\subsection{Cellular Uptake}

According to previously recorded protocols [37-40], a cellular uptake assay was performed. In 6-well plate at $1 \times 10^{4}$ A549 and HEL-299 lung cancer cells were pictured confocal laser scanning microscopy (LSM 510 Meta; Zeiss, Germany). Lysotracker Naringin-NPs with Dil (Molecular Probe, USA) stained the cell lysosome (red color), then DAPI stained the nuclei. Images were examined by confocal laser scanning microscopy (CLSM) utilizing the image analyzer software manufacturer's protocol.

\subsection{In vitro drug release profiles}

The controlled cumulative release of the Naringin and Naringin-NPs have been assessed using membrane dialysis (Spectrum Laboratory, $14 \mathrm{kDa}$-molecular weight). In short, $100 \mu \mathrm{g} / \mathrm{mL}$ of Naringin-NPs (Naringin concentration) is dialyzed at $37^{\circ} \mathrm{C}$ to $20 \mathrm{~mL}$ of phosphate buffer solutions containing Tween 80 $(0.2 \%)$ at $\mathrm{pH} 7.4$. The releasing medium was obtained by constant mild stirring $37^{\circ} \mathrm{C}$ in an orbital shaker incubator (100 RPM) at and an equivalent amount of supplemented fresh medium [40-42].

\subsection{Acridine orange and ethidium bromide (AO/EB) and Hoechst 33344 staining}


AO/EB and Hoechst 33344 staining fluorescent dyes which bound strongly to the chromatin and subsequently shine more fluorescent upon bounding. Apoptotic morphological improvements upon treating with $\mathrm{IC}_{50}$ concentrations of Naringin and Naringin-NPs against A549 and HEL-299 cancer cell lines were assessed. After staining, the cells at $20 \mathrm{X}$ magnification were displayed under a fluorescence microscopy (Olympus IX81, Germany) [43-46].

\subsection{Flow cytometry techniques}

Flow cytometry was completed to study modes of cell death over FITC-Annexin $\mathrm{V}$ and propidium iodide (PI) based apoptosis detection assay kit (Invitrogen, Thermo Fischer Scientific, USA) followed by previously reported protocol [47-49]. The A549 and HEL-299 cells were through the trypsinization to avoid the cell death. Trypsinized the cells and twice washed with PBS. The cells supernatant was removed and the cell pellets was suspended in a $250 \mu \mathrm{L} 1 \times$ binding buffer (from the kit) with $5 \mu \mathrm{L}$ of FITC Annexin-V and PI was added and incubated with $37^{\circ} \mathrm{C}$ in dark conditions. After 10 minutes, the samples were subjected to flow cytometry analysis using FACS Canto TM II, BD Biosciences.

\subsection{Hemocompatibility assay}

Human blood samples were attained from the Pulmonary and Critical Care Medicine, the First People's Hospital of Wenling, Wenling-317500, China. Red blood cells (RBCs) were collected by centrifuging the samples at $1800 \mathrm{rpm}$ at $5^{\circ} \mathrm{C}$ for $5 \mathrm{~min}$. RBCs is washed three times with PBS and suspended in $4 \mathrm{ml}$ of PBS. Next, $0.1 \mathrm{~mL}$ of diluted RBCs is applied to the Naringin and Naringin-NPs in $0.5 \mathrm{ml}$ PBS suspension

at the corresponding concentrations and incubated for $4 \mathrm{~h}$. After incubation, the samples were moved to 96-well plates. Hemolytic activity was calculated by calculation at an absorbance of $570 \mathrm{~nm}$. Monitor samples of the buffer lyses and 100 percent buffer lyses were also analyzed in these experimental procedures [50-52]. The proportion of hemolysis was determined as follows:

$\%$ hemolysis $=(A s-A n) /(A p-A n) \times 100 \%$

Whereas denotes "the absorbance of Naringin and Naringin-NPs at various concentrations $(5,10,15)$, " and An and Ap denote the negative and positive controls, respectively.

\section{Results And Discussion}

\subsection{Fabrication and characterisation of Naringin}

Figure 1 demonstrates the complete synthetic procedure. Naringin analogs conjugated with a number of successive groups at the hydroxyl terminal ends demonstrate improved stability in cellular membrane due to the dehydrogenation purpose. In addition, LA hydrophilic regulator is used for the development of polymeric prodrugs. In this work, we planned and produced the Naringin analogs for the LA polymer chains to render the Naringin -LA prodrug (Fig. 1). Conjugation was achieved by coupling Naringin to LA under the structure of Naringin -LA, and the Naringin -LA prodrugs were filtered using thin layer chromatography (TLC) and column chromatography approaches with the high yield (80.5\%). The 
lipophilic design of the drug delivery systems, which create nano-assemblies, could be synthesized in water by nanoassembly approaches without the addition of any surfactants. In addition, the organic phases of the Naringin -LA prodrugs are absorbed in the solution of DMSO into the DD-H2O aqueous suspensions. More importantly, the elimination of excess organic solvents through double distilled water from the fabricated nanoparticles rather than prodrug accumulation. In addition, TEM observations were conducted to determine the structure of supramolecular nanoassembly Naringin-NPs (Fig. 2A and B). The results of the supramolecular nanoassembly Naringin-NPs showed that the well-structured form with a sphere-shaped was $~ 80.3 \pm 3.12 \mathrm{~nm}$ (Fig. 2B). In addition, the hydrodynamic parameter of the nanoassembly Naringin-NPs was $82.7 \pm 2.05 \mathrm{~nm}$ with less poly dispersive index (PDI), as shown by the DLS method (Fig. 2C). Admirable findings are associated with the formations of supramolecular nanoassembly Naringin-NPs. The stability of the Naringin, and Naringin-NPs in PBS media was observed by measuring the particle size of the Naringin, and Naringin-NPs by dynamic light scattering. Polyplexes index (PDI), precisely Naringin, and Naringin-NPs, at an nanoparticles ratio of 100:1 were organized and incubated for $30 \mathrm{~min}$ at $37^{\circ} \mathrm{C}$ in order to check broad polyplex formations (Fig. 2D-E). All the stability analysis were three times repeated.

\subsection{In vitro Naringin release profiles}

As a traditional nucleosides analog, the main shortcomings of Naringin as a chemotherapeutic agents contain half-life small plasma and fast cytokine deaminase deactivation. We also developed supramolecular nanoassembly Naringin-NPs which could help as a reservoir to keep drugs safe and avoid the release of drugs into the general blood circulation, thereby slowing down Naringin authorization from the human body condition. Observation of the controlled release of Naringin-NPs by dialyzing against PBS at RT. As seen in Fig. 2F, Naringin free was rapidly released from Naringin-NPs and plateaued at $90.2 \pm 2.9$ compound releases after 25 hrs of incubation. In the other hand, Naringin-NPs demonstrated continuous drug release. Our findings of in vitro drug leasing revealed a sluggish inhibition of the release of free Naringin kinetics from the Naringin-NPs, which is useful to enhance the half-life blood plasma of free Naringin and enhancing drug delivery to cancer cells.

\subsection{In vitro proliferation assay by MTT}

After efficient fabrication of Naringin-NPs, MTT experiments were conducted to determine the proliferation of Naringin and Naringin-NPs of lung carcinoma cells, with A549 and HEL-299cancer cells. After treating with drugs for $24 \mathrm{hrs}$, the proliferation of the A549 and HEL-299 cells was controlled and the dose dependent curvature shows the half inhibitory concentrations $\left(\mathrm{IC}_{50}\right)$ (Fig. 3). Positively, associated to the free Naringin, supramolecular nanoassembly displayed significantly increased cytotoxic effects in both lung cancer cell lines tested. In half-inhibitory concentrations of $A 549$ cells, the $I_{50}$ is $11.12 \pm 5.09$ and $6.13 \pm 1.29$ for Naringin and Naringin-NPs respectively. In HEL-299 cells, the $\mathrm{IC}_{50}$ is $15.87 \pm 2.18$, and $5.16 \pm 2.80$ for Corilagin and Corilagin-NPs, respectively. In addition, we examined the selectivity of the Naringin and Naringin-NPs shows remarkable proliferation against the CaSki and HeLa cells (Fig. 3C). The Naringin-NPs shows remarkable proliferation against the A549 and HEL-299 cells which may be due to the presence of the hydrophilic linoleic acid of $\pi$-conjugation chain. This may be attributed due to the 
hydrophobic chain of the linoleic acid which may efficiently penetrate the cell membranes. Several attempts will be made to establish the small molecule nano-assembled nanoparticles for promising anticancer agents by introducing the various lipophilic spacers on the potential small molecules.

\subsection{Cellular uptake efficiency of Naringin-NPs}

We cross-linked that the Naringin-NPs make it possible to interact with A549 and HEL-299lungtumor cells and ions, thereby enhancing intracellular absorption into the lung cancer cell. A confocal laser scanning microscopy (LCSM) was used to test the localizations of subcellular of Naringin-NPs in the A549 and HEL-299 lung cancer cell lines (Fig. 4). In the concentration $20 \mathrm{nM}$ with Naringin-NPs with different incubation times $(5,10,20$ and $30 \mathrm{~min}$ ) were labelled with the Dil fluorescence tracker (red color) for the interpretations, for this comparison the cells lysosome and the nucleus was labeled with the DAPI (blue color). The new yellow color fluorescents were paired with Dil, and DAPI fluorescence in the A549 and HEL-299 lung cancer cell line, whereas Naringin-NPs could be confined with lysosome during internalization.

\subsection{Morphological examination by acridine orange/ethidium bromide (AO/EB)}

Naringin and Naringin-NPs were tested using a fluorescent microscopic examination of acridine orange/ ethidium bromide (AO/EB) stained in A549 and HEL-299 lung cancer cell lines indicative of morphological changes (Fig. 5). Usually nanoparticles causes cell death through the apoptosis and necrosis pathways. Ironically, after 24-hour treatment with their $\mathrm{IC}_{50}$ concentration of the Naringin and Naringin-NPs, Naringin-NPs display a higher proportion of cell death by apoptosis pathway than free Naringin treatments.

\subsection{Morphological examination by Hoechst-33258 staining}

Naringin and Naringin-NPs were tested using a fluorescent microscopic examination of Hoechst-33258 staining (nuclear staining) stained in A549 and HEL-299 lung cancer cell lines indicative of morphological changes (Fig. 6). Usually nanoparticles causes cell death through the apoptosis and necrosis pathways. Ironically, after 24-hour treatment with their $\mathrm{IC}_{50}$ concentration of the Naringin and Naringin-NPs, Naringin-NPs display a higher proportion of cell death by apoptosis pathway than free Naringin treatments.

\subsection{Flow cytometry-confirmation of the apoptosis}

Apoptosis can be used as a significant impairment to the growth of a tumor cells. The primary qualitative screening methods of the AO/EB and nuclear staining methods clearly shows the supramolecular nanoassembly induce the cell death through apoptosis mode. In this quantitative experiments displays the number of cancer cell death by the flow cytometry via staining of the FITC Annexin-V and propidium iodide (PI) in the A549 and HEL-299 lung cancer cells. Naringin and Naringin-NPs were tested using a FITC Annexin- $V$ with the propidium iodide (PI) examination in A549 and HEL-299 lung cancer cell lines 
(Fig. 7). Ironically, after 24-hour treatment with their $\mathrm{IC}_{50}$ concentration of the Naringin and Naringin-NPs, Naringin-NPs with high percentage of the apoptosis than free Naringin.

\subsection{Hemocompatibility assay}

Analysis of the relationship between nanoparticles and human blood erythrocytes using hemolysis assays is crucial to evaluating the blood viability of nanoparticles (Fig. 8). Free Naringin and NaringinNPs have been shown to have excellent biocompatibility with human red blood cells, as shown in Fig. 8 . The function of toxic substances appears to be nano-specific. According to IOS/Technical Report 7406, the hemolytic levels of nanoparticles or materials is limited to $5 \%$. The release of erythrocytes by Naringin-NPs was insignificant, suggesting that the nanoparticles had a marginal amount of toxicity and were thus safe for the cells.

\section{Conclusion}

We have effectively engineered and illustrated the lipophilic and fast metabolic Naringin prodrugs for a more pharmacologically effective nano prodrug. We find out that supramolecular nanoassembly of Naringin nanoparticles display improved controlled drug release and enhanced extracellular environment uptake to possibly resolve cancer cell aggregations by EPR. After a successful development, we tested the MTT of Naringin and Naringin-NPs against A549 and HEL-299 lung tumor cell lines. In addition, the morphological changes experiments such as acridine orange/ethidium bromide (AO-EB), Hoechst-33258 staining results shows that the supramolecular nanoassembly of Naringin nanoparticles induce apoptosis in lung cancer cells. Further, we have confirmed the apoptosis by flowcytometry techniques. The findings of Naringin-NPs nanoparticles will invention possible application of lung cancer patents for better chemotherapy and potential clinical investigations.

\section{Declarations}

\section{Conflict of the interest}

None

\section{References}

1. Ghosh S, Lalani R, Maiti K, Banerjee S, Bhatt H, Bobde YS, Patel V, Biswas S, Bhowmick S, Misra A (2021) Synergistic co-loading of vincristine improved chemotherapeutic potential of pegylated liposomal doxorubicin against triple negative breast cancer and non-small cell lung cancer. Nanomedicine Nanotechnology Biol Med 31:102320. https://doi.org/https://doi.org/10.1016/j.nano.2020.102320

2. Novoselova MV, Loh HM, Trushina DB, Ketkar A, Abakumova TO, Zatsepin TS, Kakran M, Brzozowska AM, Lau HH, Gorin DA, Antipina MN, Brichkina Al (2020) Biodegradable Polymeric Multilayer 
Capsules for Therapy of Lung Cancer. ACS Appl Mater Interfaces 12:5610-5623.

https://doi.org/10.1021/acsami.9b21381

3. Abdelaziz HM, Elzoghby AO, Helmy MW, Abdelfattah E-ZA, Fang J-Y, Samaha MW, Freag MS (2020) Inhalable Lactoferrin/Chondroitin-Functionalized Monoolein Nanocomposites for Localized Lung Cancer Targeting, ACS Biomater. Sci Eng 6:1030-1042.

https://doi.org/10.1021/acsbiomaterials.9b01639

4. Xie X, Li Y, Zhao D, Fang C, He D, Yang Q, Yang L, Chen R, Tan Q, Zhang J (2020) Oral administration of natural polyphenol-loaded natural polysaccharide-cloaked lipidic nanocarriers to improve efficacy against small-cell lung cancer. Nanomedicine Nanotechnology Biol Med 29:102261.

https://doi.org/https://doi.org/10.1016/j.nano.2020.102261

5. Guan X, Yang B, Xie M, Ban DK, Zhao X, Lal R, Zhang F (2019) MRI reporter gene MagA suppresses transferrin receptor and maps Fe2 + dependent lung cancer, Nanomedicine Nanotechnology. Biol Med 21:102064. https://doi.org/https://doi.org/10.1016/j.nano.2019.102064

6. Chen Y, Sun J, Huang Y, Liu Y, Liang L, Yang D, Lu B, Li S (2019) Targeted codelivery of doxorubicin and IL-36y expression plasmid for an optimal chemo-gene combination therapy against cancer lung metastasis. Nanomedicine Nanotechnology Biol Med 15:129-141.

https://doi.org/https://doi.org/10.1016/j.nano.2018.09.005

7. Zhang L, Li J, Hao C, Guo W, Wang D, Zhang J, Zhao Y, Duan S, Yao W (2018) Up-regulation of exosomal miR-125a in pneumoconiosis inhibits lung cancer development by suppressing expressions of EZH2 and hnRNPK. RSC Adv 8:26538-26548. https://doi.org/10.1039/C8RA03081B

8. Amreddy N, Babu A, Panneerselvam J, Srivastava A, Muralidharan R, Chen A, Zhao YD, Munshi A, Ramesh R (2018) Chemo-biologic combinatorial drug delivery using folate receptor-targeted dendrimer nanoparticles for lung cancer treatment. Nanomedicine Nanotechnology Biol Med 14:373-384. https://doi.org/https://doi.org/10.1016/j.nano.2017.11.010

9. Zhang C, Li C, Xu Y, Feng L, Shang D, Yang X, Han J, Sun Z, Li Y, Li X (2015) Integrative analysis of lung development-cancer expression associations reveals the roles of signatures with inverse expression patterns. Mol Biosyst 11:1271-1284. https://doi.org/10.1039/C5MB00061K

10. Liu Z, Ma L, Wen Z-S, Cheng Y-X, Zhou G-B (2014) Ethoxysanguinarine Induces Inhibitory Effects and Downregulates CIP2A in Lung Cancer Cells, ACS Med. Chem Lett 5:113-118.

https://doi.org/10.1021/ml400341k

11. Chen Q, Jiao D, Wu Y, Wang L, Hu H, Song J, Yan J, Wu L (2013) Functional and pathway enrichment analysis for integrated regulatory network of high- and low-metastatic lung cancer. Mol Biosyst 9:3080-3090. https://doi.org/10.1039/C3MB70288J

12. Sung H-J, Ahn J-M, Yoon Y-H, Rhim T-Y, Park C-S, Park J-Y, Lee S-Y, Kim J-W, Cho J-Y (2011) Identification and Validation of SAA as a Potential Lung Cancer Biomarker and its Involvement in Metastatic Pathogenesis of Lung Cancer. J Proteome Res 10:1383-1395.

https://doi.org/10.1021/pr101154j 
13. Wu Y, Crawford M, Yu B, Mao Y, Nana-Sinkam SP, Lee LJ (2011) MicroRNA Delivery by Cationic Lipoplexes for Lung Cancer Therapy. Mol Pharm 8:1381-1389. https://doi.org/10.1021/mp2002076

14. Keller A, Backes C, Leidinger P, Kefer N, Boisguerin V, Barbacioru C, Vogel B, Matzas M, Huwer H, Katus HA, Stähler C, Meder B, Meese E (2011) Next-generation sequencing identifies novel microRNAs in peripheral blood of lung cancer patients. Mol BioSyst 7:3187-3199. https://doi.org/10.1039/C1MB05353A

15. Magee ND, Villaumie JS, Marple ET, Ennis M, Elborn JS, McGarvey JJ (2009) Ex Vivo Diagnosis of Lung Cancer Using a Raman Miniprobe. J Phys Chem B 113:8137-8141. https://doi.org/10.1021/jp900379w

16. Park H-J, Kim B-G, Lee S-J, Heo S-H, Kim J-Y, Kwon T-H, Lee E-B, Ryoo H-M, Cho J-Y (2008) Proteomic Profiling of Endothelial Cells in Human Lung Cancer. J Proteome Res 7:1138-1150. https://doi.org/10.1021/pr7007237

17. Li P, Wu H, Wang Y, Peng W, Su W (2020) Toxicological evaluation of naringin: Acute, subchronic, and chronic toxicity in Beagle dogs. Regul Toxicol Pharmacol 111:104580. https://doi.org/https://doi.org/10.1016/j.yrtph.2020.104580

18. Gollavilli H, Hegde AR, Managuli RS, Bhaskar KV, Dengale SJ, Reddy MS, Kalthur G, Mutalik S (2020) Naringin nano-ethosomal novel sunscreen creams: Development and performance evaluation. Colloids Surfaces B Biointerfaces 193:111122. https://doi.org/https://doi.org/10.1016/j.colsurfb.2020.111122

19. Syed AA, Reza MI, Shafiq M, Kumariya S, Singh P, Husain A, Hanif K, Gayen JR (2020) Naringin ameliorates type 2 diabetes mellitus-induced steatohepatitis by inhibiting RAGE/NF-KB mediated mitochondrial apoptosis. Life Sci 257:118118.

https://doi.org/https://doi.org/10.1016/j.Ifs.2020.118118

20. Gerçek E, Zengin H, Erdem Erişir F, Yılmaz Ö (2021) Biochemical changes and antioxidant capacity of naringin and naringenin against malathion toxicity in Saccharomyces cerevisiae. Comp Biochem Physiol Part C Toxicol Pharmacol 241:108969.

https://doi.org/https://doi.org/10.1016/j.cbpc.2020.108969

21. Guo X, Li K, Guo A, Li E (2020) Intestinal absorption and distribution of naringin, hesperidin, and their metabolites in mice. J Funct Foods 74:104158.

https://doi.org/https://doi.org/10.1016/j.jff.2020.104158

22. Ni K, Guo J, Bu B, Pan Y, Li J, Liu L, Luo M, Deng L, Naringin as a plant-derived bitter tastant promotes proliferation of cultured human airway epithelial cells via activation of TAS2R signaling, Phytomedicine. 84 (2021) 153491. https://doi.org/https://doi.org/10.1016/j.phymed.2021.153491

23. Tang X, Zhao H, Jiang W, Zhang S, Guo S, Gao X, Yang P, Shi L, Liu L (2018) Pharmacokinetics and pharmacodynamics of citrus peel extract in lipopolysaccharide-induced acute lung injury combined with Pinelliae Rhizoma Praeparatum. Food Funct 9:5880-5890.

https://doi.org/10.1039/C8F001337C 
24. Dai H, Yang C, Ma X, Lin Y, Chen G (2011) A highly sensitive and selective sensing ECL platform for naringin based on $\beta$-Cyclodextrin functionalized carbon nanohorns. Chem Commun 47:1191511917. https://doi.org/10.1039/C1CC14611D

25. Feng X, Wu T, Yu B, Wang Y, Zhong S (2017) Hydrophilic surface molecularly imprinted naringin prepared via reverse atom transfer radical polymerization with excellent recognition ability in a pure aqueous phase. RSC Adv 7:28082-28091. https://doi.org/10.1039/C7RA00202E

26. Jabbari M, Khosravi N, Feizabadi M, Ajloo D (2017) Solubility temperature and solvent dependence and preferential solvation of citrus flavonoid naringin in aqueous DMSO mixtures: an experimental and molecular dynamics simulation study. RSC Adv 7:14776-14789. https://doi.org/10.1039/C7RA00038C

27. Selvaraj S, Krishnaswamy S, Devashya V, Sethuraman S, Krishnan UM (2014) Investigations on the membrane interactions of naringin and its complexes with copper and iron: implications for their cytotoxicity. RSC Adv 4:46407-46417. https://doi.org/10.1039/C4RA08157A

28. Mandial D, Khullar P, Kumar H, Ahluwalia GK, Bakshi MS (2018) Naringin-Chalcone BioflavonoidProtected Nanocolloids: Mode of Flavonoid Adsorption, a Determinant for Protein Extraction. ACS Omega 3:15606-15614. https://doi.org/10.1021/acsomega.8b01776

29. Shao Y, You D, Lou Y, Li J, Ying B, Cheng K, Weng W, Wang H, Yu M, Dong L (2019) Controlled Release of Naringin in GelMA-Incorporated Rutile Nanorod Films to Regulate Osteogenic Differentiation of Mesenchymal Stem Cells. ACS Omega 4:19350-19357. https://doi.org/10.1021/acsomega.9b02751

30. Yu M, You D, Zhuang J, Lin S, Dong L, Weng S, Zhang B, Cheng K, Weng W, Wang H (2017) Controlled Release of Naringin in Metal-Organic Framework-Loaded Mineralized Collagen Coating to Simultaneously Enhance Osseointegration and Antibacterial Activity. ACS Appl Mater Interfaces 9:19698-19705. https://doi.org/10.1021/acsami.7b05296

31. Wang F, Zhao C, Tian G, Wei X, Ma Z, Cui J, Wei R, Bao Y, Kong W, Zheng J (2020) Naringin Alleviates Atherosclerosis in ApoE-/- Mice by Regulating Cholesterol Metabolism Involved in Gut Microbiota Remodeling. J Agric Food Chem 68:12651-12660. https://doi.org/10.1021/acs.jafc.0c05800

32. Cao H, Liu J, Shen P, Cai J, Han Y, Zhu K, Fu Y, Zhang N, Zhang Z, Cao Y (2018) Protective Effect of Naringin on DSS-Induced Ulcerative Colitis in Mice. J Agric Food Chem 66:13133-13140. https://doi.org/10.1021/acs.jafc.8b03942

33. Zeng X, Yao H, Zheng Y, Chen T, Peng W, Wu H, Su W (2020) Metabolite Profiling of Naringin in Rat Urine and Feces Using Stable Isotope-Labeling-Based Liquid Chromatography-Mass Spectrometry. J Agric Food Chem 68:409-417. https://doi.org/10.1021/acs.jafc.9b06494

34. Ding D, Li K, Zhu Z, Pu KY, Hu Y, Jiang X, Liu B (2011) Conjugated polyelectrolyte-cisplatin complex nanoparticles for simultaneous in vivo imaging and drug tracking. Nanoscale 3:1997-2002. https://doi.org/10.1039/c0nr00950d

35. Huang Y, He Y, Huang Z, Jiang Y, Chu W, Sun X, Huang L, Zhao C (2017) Coordination self-assembly of platinum-bisphosphonate polymer-metal complex nanoparticles for cisplatin delivery and 
effective cancer therapy. Nanoscale 9:10002-10019. https://doi.org/10.1039/c7nr02662e

36. Han W, Shi L, Ren L, Zhou L, Li T, Qiao Y, Wang H (2018) A nanomedicine approach enables codelivery of cyclosporin A and gefitinib to potentiate the therapeutic efficacy in drug-resistant lung cancer, Signal Transduct. Target Ther 3:1-10. https://doi.org/10.1038/s41392-018-0019-4

37. Parker JP, Ude Z, Marmion CJ (2016) Exploiting developments in nanotechnology for the preferential delivery of platinum-based anti-cancer agents to tumours: Targeting some of the hallmarks of cancer. Metallomics 8:43-60. https://doi.org/10.1039/c5mt00181a

38. Llinàs MC, Martínez-Edo G, Cascante A, Porcar I, Borrós S, Sánchez-García D (2018) Preparation of a mesoporous silica-based nano-vehicle for dual DOX/CPT ph-triggered delivery. Drug Deliv 25:11371146. https://doi.org/10.1080/10717544.2018.1472678

39. Tambe P, Kumar P, Paknikar KM, Gajbhiye V (2018) Decapeptide functionalized targeted mesoporous silica nanoparticles with doxorubicin exhibit enhanced apoptotic effect in breast and prostate cancer cells. Int J Nanomedicine 13:7669-7680. https://doi.org/10.2147/IJN.S184634

40. Deng B, Ma P, Xie Y (2015) Reduction-sensitive polymeric nanocarriers in cancer therapy: a comprehensive review. Nanoscale 7:12773-12795. https://doi.org/10.1039/c5nr02878g

41. Kim J, Pramanick S, Lee D, Park H, Kim WJ (2015) Polymeric biomaterials for the delivery of platinum-based anticancer drugs. Biomater Sci 3:1002-1017. https://doi.org/10.1039/c5bm00039d

42. Tabatabaei Rezaei SJ, Amani V, Nabid MR, Safari N, Niknejad H (2015) Folate-decorated polymeric Pt(ii) prodrug micelles for targeted intracellular delivery and cytosolic glutathione-triggered release of platinum anticancer drugs. Polym Chem 6:2844-2853. https://doi.org/10.1039/c5py00156k

43. Mohamed Subarkhan MK, Ramesh R, Liu Y (2016) Synthesis and molecular structure of arene ruthenium(ii) benzhydrazone complexes: impact of substitution at the chelating ligand and arene moiety on antiproliferative activity. New J Chem 40:9813-9823.

https://doi.org/10.1039/C6NJ01936F

44. Li X, Gao Y (2020) Synergistically fabricated polymeric nanoparticles featuring dual drug delivery system to enhance the nursing care of cervical cancer. Process Biochem 98:254-261. https://doi.org/https://doi.org/10.1016/j.procbio.2020.09.010

45. Kasibhatla S, Amarante-Mendes GP, Finucane D, Brunner T, Bossy-Wetzel E, Green DR, Acridine Orange/Ethidium Bromide (AO/EB) Staining to Detect Apoptosis, CSH Protoc (2006) (2006) 799803. https://doi.org/10.1101/pdb.prot4493

46. Mohamed Kasim MS, Sundar S, Rengan R (2018) Synthesis and structure of new binuclear ruthenium(ii) arene benzil bis(benzoylhydrazone) complexes: investigation on antiproliferative activity and apoptosis induction. Inorg Chem Front 5:585-596. https://doi.org/10.1039/C7QI00761B

47. Zhang W-Y, Wang Y-J, Du F, He M, Gu Y-Y, Bai L, Yang L-L, Liu Y-J (2019) Evaluation of anticancer effect in vitro and in vivo of iridium(III) complexes on gastric carcinoma SGC-7901 cells. Eur J Med Chem 178:401-416. https://doi.org/https://doi.org/10.1016/j.ejmech.2019.06.003

48. Liu K, Liu P, Liu R, Wu X (2015) Dual AO/EB staining to detect apoptosis in osteosarcoma cells compared with flow cytometry. Med Sci Monit Basic Res 21:15-20. 
https://doi.org/10.12659/MSMBR.893327

49. Sathiya Kamatchi T, Mohamed Subarkhan MK, Ramesh R, Wang H, Małecki JG (2020) Investigation into antiproliferative activity and apoptosis mechanism of new arene Ru(ii) carbazole-based hydrazone complexes. Dalt Trans 49:11385-11395. https://doi.org/10.1039/D0DT01476A

50. Pragathiswaran C, Smitha C, Barabadi H, Al-Ansari MM, Al-Humaid LA, Saravanan M (2020) TiO2@ZnO nanocomposites decorated with gold nanoparticles: Synthesis, characterization and their antifungal, antibacterial, anti-inflammatory and anticancer activities. Inorg Chem Commun 121:108210. https://doi.org/https://doi.org/10.1016/j.inoche.2020.108210

51. Fischer D, Li Y, Ahlemeyer B, Krieglstein J, Kissel T (2003) In vitro cytotoxicity testing of polycations: influence of polymer structure on cell viability and hemolysis. Biomaterials 24:1121-1131. https://doi.org/https://doi.org/10.1016/S0142-9612(02)00445-3

52. Tramer F, Da Ros T, Passamonti S (2012) Screening of fullerene toxicity by hemolysis assay. Methods Mol Biol 926:203-217. https://doi.org/10.1007/978-1-62703-002-1_15

\section{Figures}

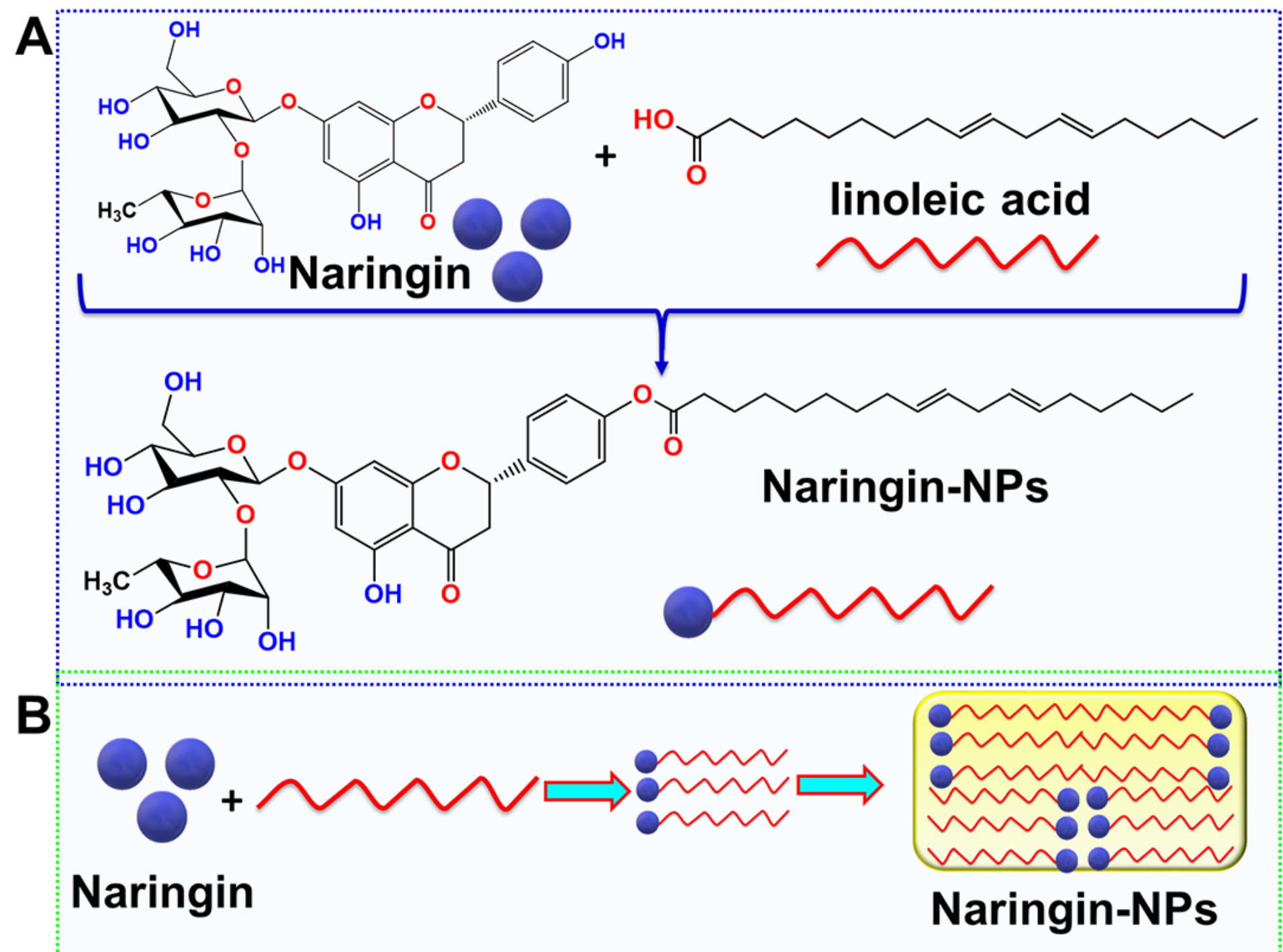


Figure 1

Graphical description of Naringin-NPs affecting lung cancer cells. (a) Structure of the Naringin. Graphic design of the Naringin prodrug formulation preparation process and can be nanoassembly in water by Naringin-NPs displaying a possible chemotherapy outcome.
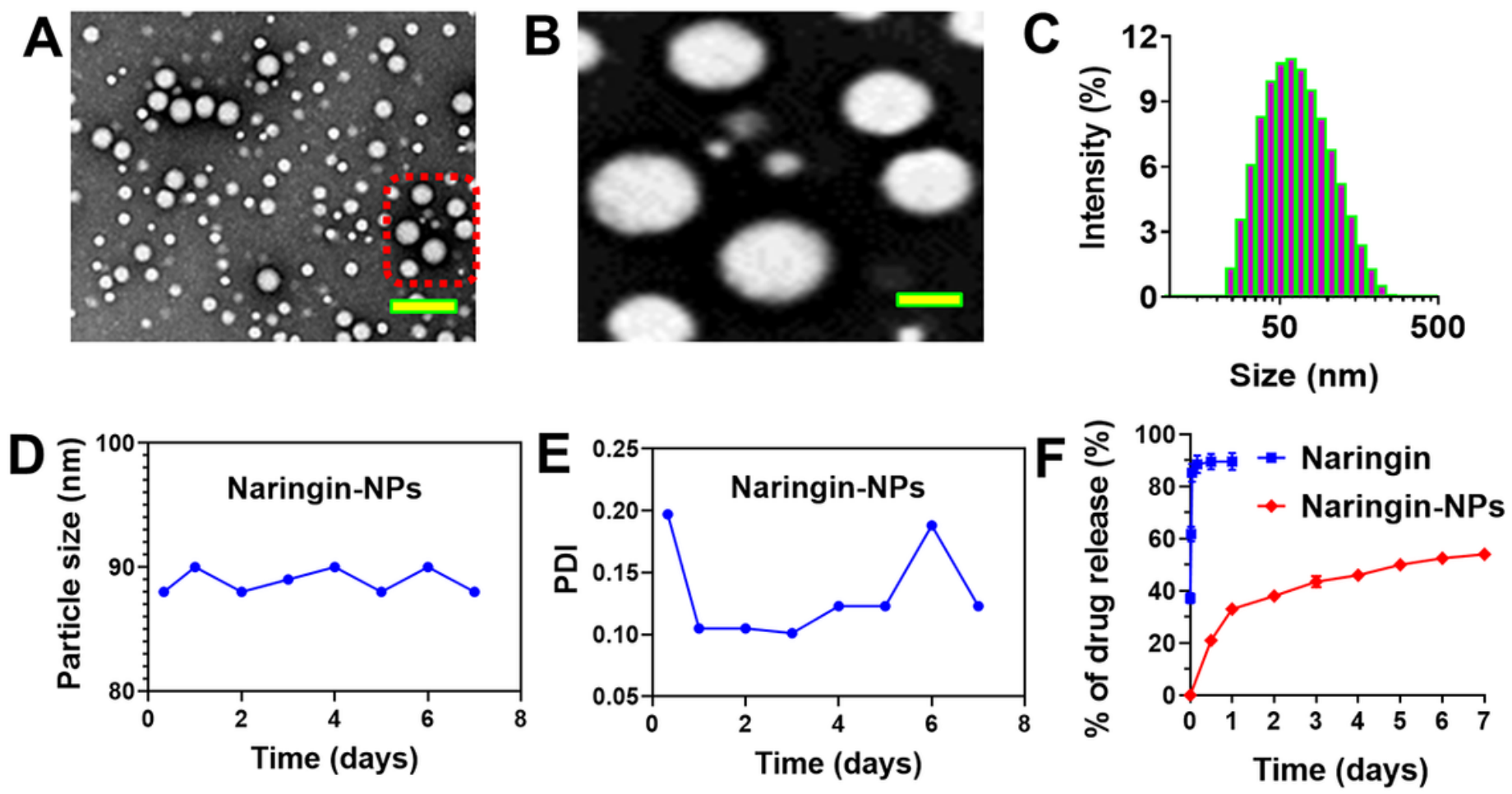

Figure 2

Characteristic assessment of Naringin-NPs. (A) TEM representation of Naringin-NPs. The scale bar is 100 nm. (B) Expand view of the TEM image. (C) Hydrodynamic parameter Naringin-NPs. (D and E) The stability of Hydrodynamic parameter diagram of Naringin-NPs to be investigated by a DLS method. F) In vitro controlled drug release profiles of Naringin drugs from Naringin-NPs. The solution contains NaringinNPs which have been dialyzed against PBS at $37^{\circ} \mathrm{C}$. 

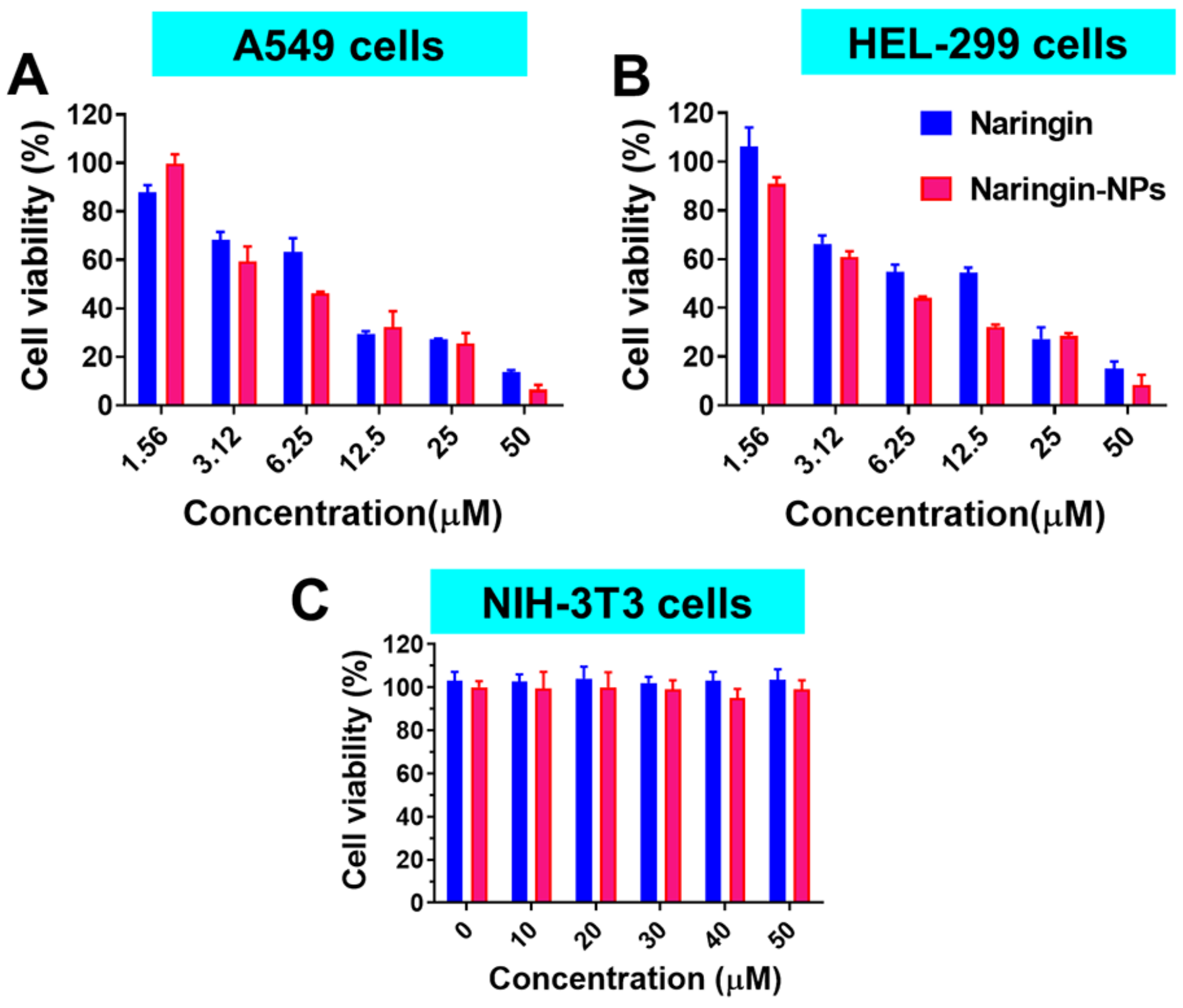

Figure 3

In vitro proliferation of A549 and HEL-299 lung cancer cell lines. MTT assay was performed to examine the proliferation of the cancer cells with different concentrations. (A and B) The proliferation of the A549 and HEL-299 lung cancer cell lines treatment with Naringin and Naringin-NPs. (C) The proliferation of the non-cancerous NIH-3T3 cell lines treatment with Naringin and Naringin-NPs. 


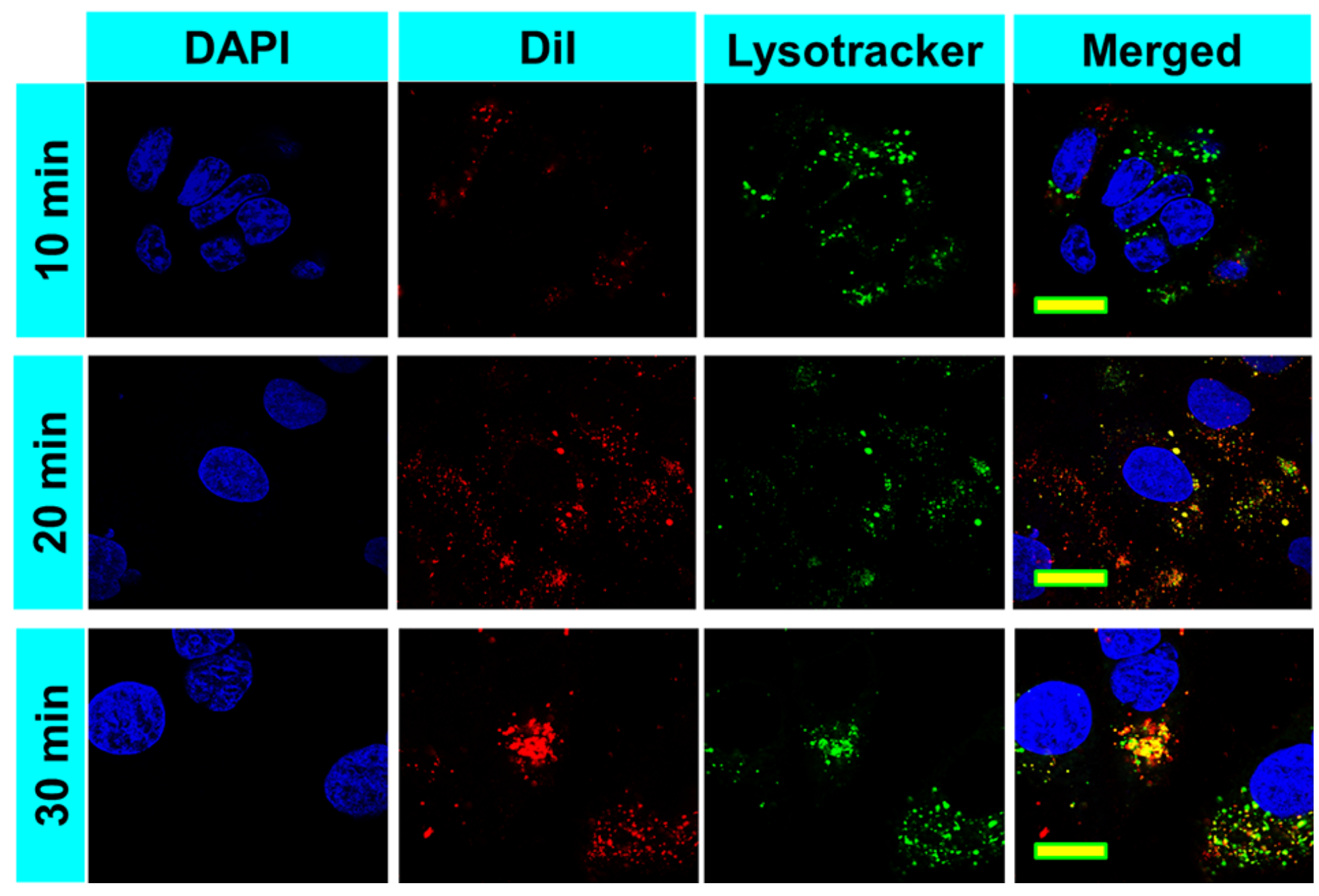

Figure 4

Cellular uptake efficiency of the A549 lung cancer cells. Lysosomes labeled with subcellular localization of Naringin-NPs in A549 lung cancer cell lines at different incubation periods. Scale bar $=20 \mu \mathrm{m}$. 

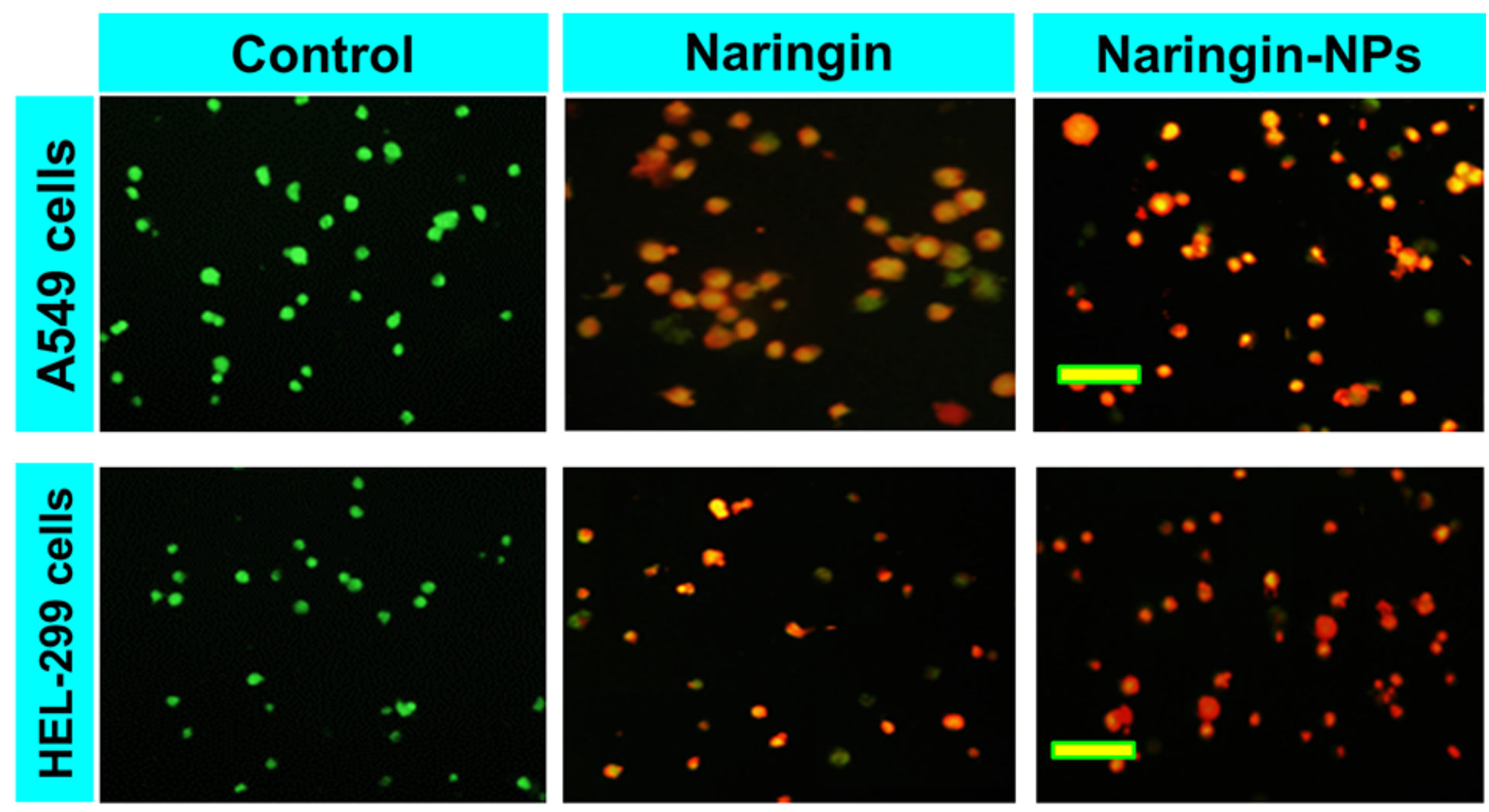

Figure 5

Dual AO/EB fluorescent staining of A549 and HEL-299 lung cancer cell lines after treating with Naringin and Naringin-NPs with IC50 concentration for 24 hrs.
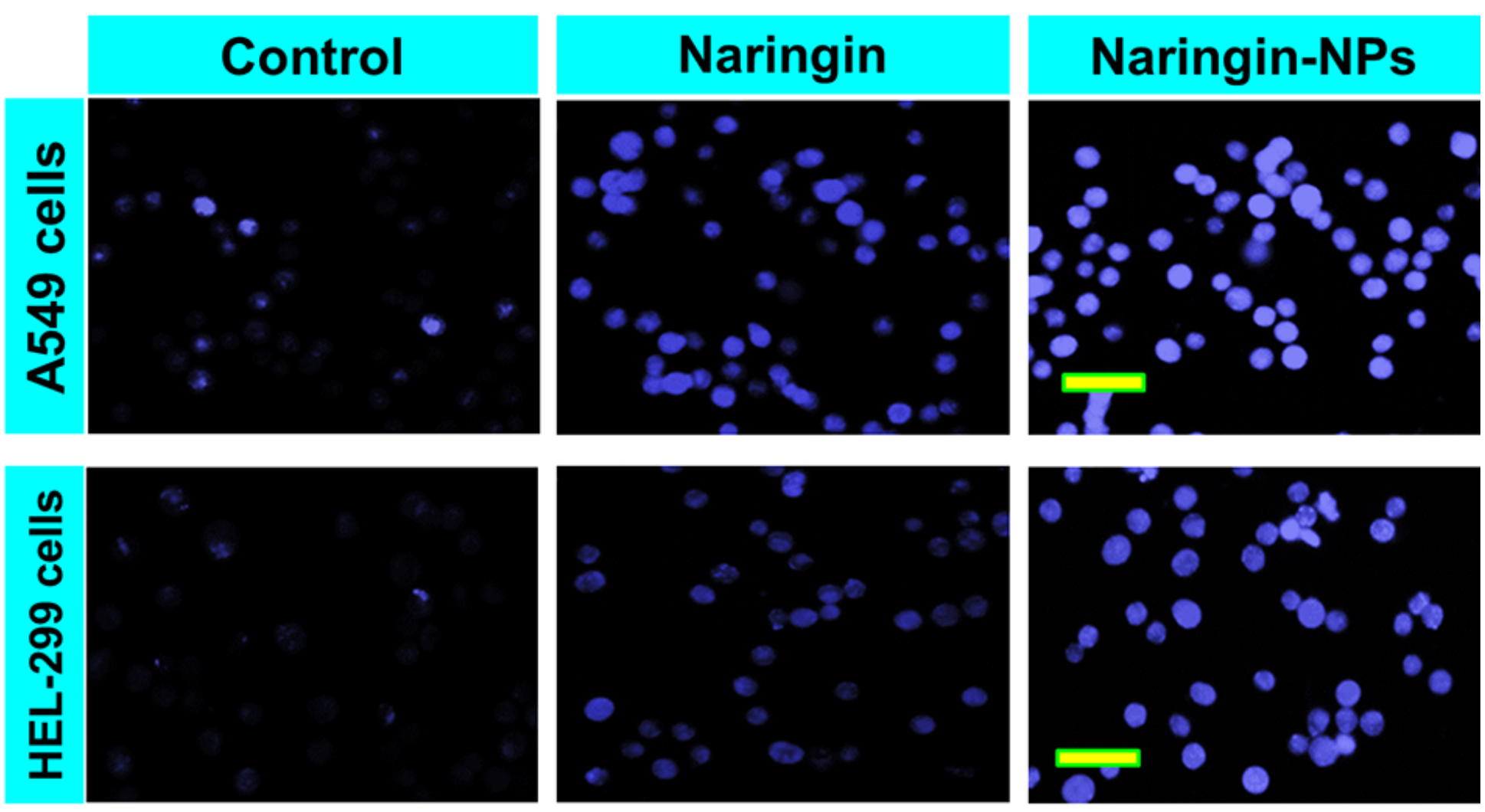

Figure 6 
Nuclear staining of A549 and HEL-299 lung cancer cell lines after treating with Naringin and Naringin-NPs with IC50 concentration for 24 hrs.
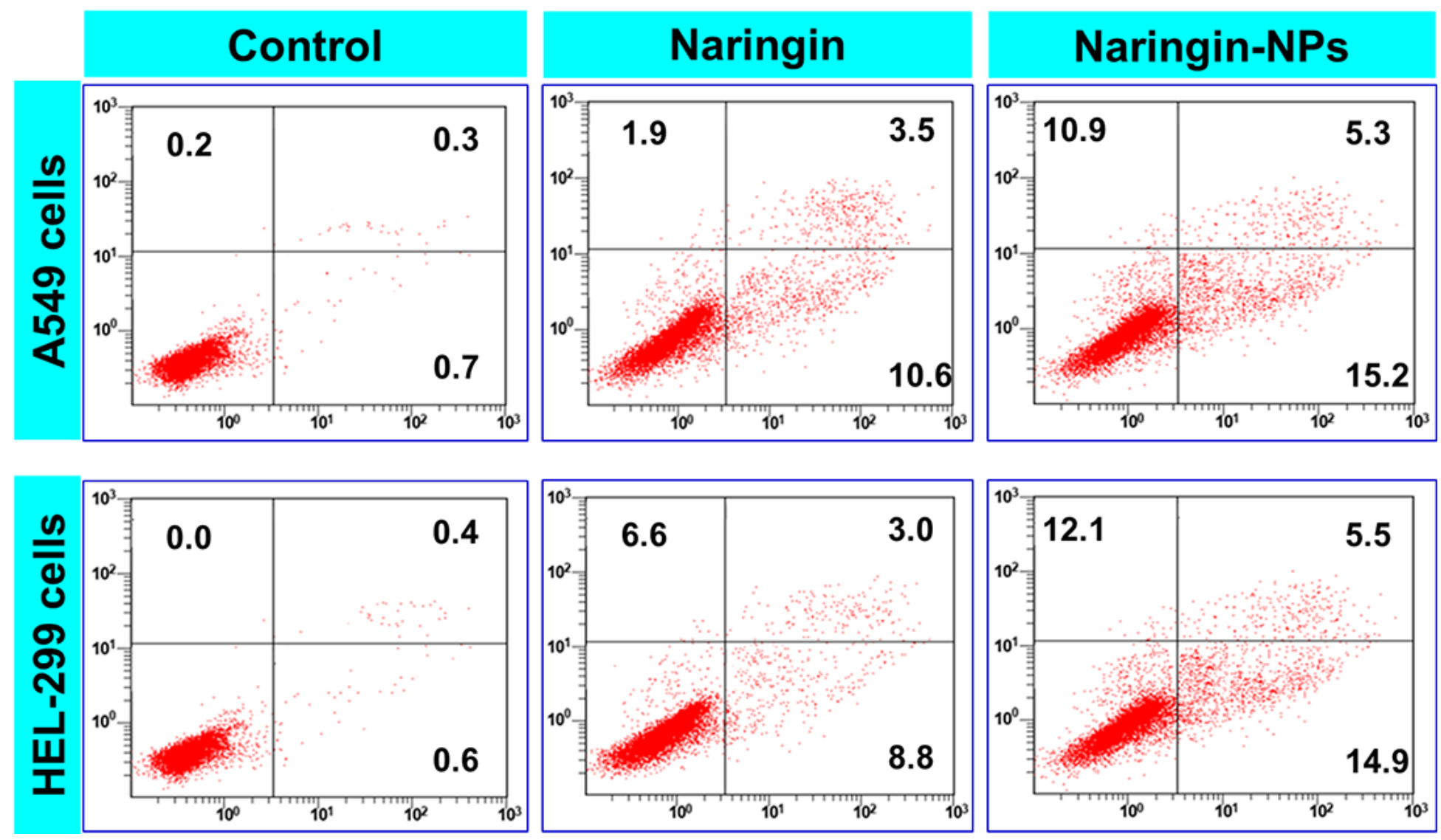

Figure 7

Flow cytometry examination were performed to confirm the apoptosis of A549 and HEL-299 lung cancer cells. The A549 and HEL-299 cells were treated with Naringin and Naringin-NPs with IC50 concentration for 24 hrs and stained by FITC-Annexin V and PI for flow cytometry examination. 

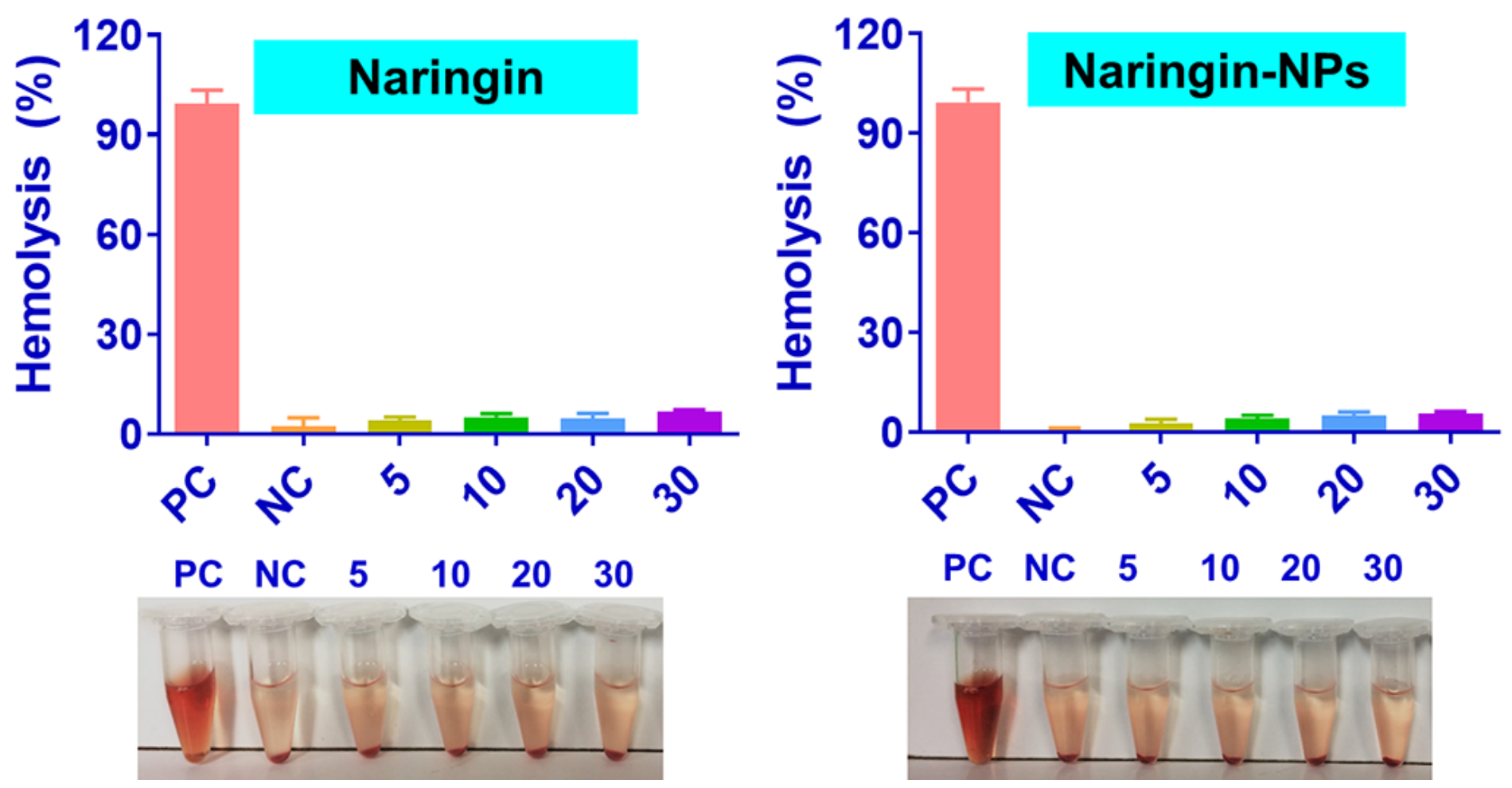

Figure 8

Hemocompatibility assay with various concentrations of Naringin and Naringin-NPs. The result of hemocompatibility assay shows that the remarkable hemocompatibility which displays that is precise bio-compatibility profiles for in vivo.

\section{Supplementary Files}

This is a list of supplementary files associated with this preprint. Click to download.

- Graphicalabstract.docx 